\title{
BMJ Open Indirect comparison between immunotherapy alone and immunotherapy plus chemotherapy as first-line treatment for advanced non- small cell lung cancer: a systematic review
}

\author{
Lingling Li (D) , ${ }^{1}$ Fei Xu, ${ }^{2}$ Yu Chen, ${ }^{1}$ Xiaoli Ren, ${ }^{3}$ Yu Liu, ${ }^{1}$ Yuan Chen, ${ }^{1}$ Shu Xia ${ }^{1}$
}

To cite: Li L, Xu F, Chen Y, et al. Indirect comparison between immunotherapy alone and immunotherapy plus chemotherapy as firstline treatment for advanced non-small cell lung cancer: a systematic review. BMJ Open 2020;10:e034010. doi:10.1136/ bmjopen-2019-034010

- Prepublication history and additional material for this paper is available online. To view these files, please visit the journal online (http://dx.doi.org/10. 1136/bmjopen-2019-034010)

Received 01 September 2019 Revised 04 August 2020 Accepted 21 September 2020

\section{Check for updates}

(C) Author(s) (or their employer(s)) 2020. Re-use permitted under CC BY-NC. No commercial re-use. See rights and permissions. Published by BMJ.

${ }^{1}$ Department of Oncology, Tongji Hospital of Tongji Medical College of Huazhong University of Science and Technology, Wuhan, China

${ }^{2}$ Department of Oncology, Affiliated Hospital of Hebei University of Engineering, Handan, China

${ }^{3}$ Department of Hematology, Hubei Provincial Hospital of Integrated Chinese and Western Medicine, Wuhan, China

Correspondence to

Dr Shu Xia;

xiashutj@hotmail.com

\section{ABSTRACT}

Objectives Use of immune checkpoint inhibitors as first-line treatment for advanced (stage IIIB/IV) nonsmall cell lung cancer (NSCLC) remains controversial. Clinical trials comparing single-drug immunotherapy (IO) with immunotherapy plus chemotherapy (IC) are lacking. We aimed to compare the efficacy of IO alone with that of IC as first-line treatment for advanced NSCLC.

Design Systematic review.

Data sources PubMed, the Cochrane Library and Embase for related studies on NSCLC; ClinicalTrials.gov, American Society of Clinical Oncology Meeting Library and World Conference on Lung Cancer for relevant conference abstracts (to July 2019).

Eligibility criteria Articles meeting the following criteria were selected: (1) randomised controlled trials on NSCLC treatment, (2) all individuals in the studies had not received treatment previously and (3) research on 10 monotherapy using programmed death-1/programmed death ligand-1 (PD-L1) inhibitors or IC.

Data extraction and synthesis After reading the original literature, two reviewers independently extracted the relevant information. The primary outcomes were progression-free survival (PFS), overall survival (OS) and objective response rate (ORR). We also extracted data on treatment-related adverse events and immune-related adverse events (irAEs).

Results Overall, 10 randomised controlled clinical trials $(n=5765)$ were included. As first-line treatment for advanced NSCLC, IC tended to yield better PFS, OS and ORR than did IO. Furthermore, IC yielded significantly better PFS than 10 when tumour PD-L1 expression was at least $50 \%$ (HR: $1.81,95 \% \mathrm{Cl}: 1.18$ to 2.78 ) and yielded a better OS and PFS when tumour PD-L1 expression was at least $1 \%$; 10 resulted in fewer adverse events than did IC. However, the incidence of irAEs was higher for 10 than for IC.

Conclusions The findings of the indirect comparison indicate that IC as first-line treatment for advanced NSCLC is significantly more effective than 10 in patients with PD-L1 expression in at least $50 \%$ of tumour cells. Trial registration number CRD 42018116589.
Strengths and limitations of this study

- This study examined published articles and conference reports of clinical trials to indirectly compare the efficacy of single-drug immunotherapy with that of immunotherapy plus chemotherapy as first-line treatment for advanced non-small cell lung cancer (NSCLC).

- According to different programmeddeath ligand-1 expression, we performed subgroup analyses of the therapeutic effects between single-drug immunotherapy and immunotherapy plus chemotherapy as first-line treatment for advanced NSCLC.

- In this study, we also performed a subgroup analysis of immune-related adverse events and adverse events of interest in the as-treated population.

- There are few randomised controlled trials on firstline treatment for advanced NSCLC with the current findings; thus, few studies were included in the current study, and further analysis was not possible.

- Three of the included studies were summaries of conference abstracts, some of which did not have final results.

\section{INTRODUCTION}

Lung cancer is among the malignancies with the highest incidence and mortality rates worldwide. ${ }^{1}$ Among the subtypes, nonsmall cell lung cancer (NSCLC) is the most common, accounting for $85 \%$ of all lung malignancies and has a poor prognosis, with a 5-year survival rate of only $15 \%$. $^{2}$ Platinumbased chemotherapy as first-line treatment for epidermal growth factor receptor (EGFR) mutation-negative or anaplastic lymphoma kinase (ALK) translocation-negative NSCLC has yielded unsatisfactory results, ${ }^{3}$ and thus, other treatment options have been considered. Breakthroughs in immune checkpoint inhibitors (ICIs) therapy have made ICIs a better choice for NSCLC treatment. ${ }^{4-6}$ Among ICIs, the most prominent representatives are 
programmed death 1 (PD-1) inhibitors and programmed death ligand 1 (PD-L1) inhibitors. ${ }^{4}$ PD-1/PD-L1 inhibitors activate the immune system to attack tumour cells by blocking the binding of PD-L1/PD-L2 on the surface of tumour cells to PD-1 on the surface of T cells. ${ }^{78}$

In the KEYNOTE-024 study, ${ }^{9}$ open-label, phase III trial, in a 1:1 ratio, enrolled 305 patients with previously untreated advanced NSCLC and PD-L1 expression in at least $50 \%$ of tumour cells. The patients were randomly assigned to pembrolizumab and platinum-based chemotherapy. The median progression-free survival (PFS) was 10.3 months in the pembrolizumab group and 6.0 months in the chemotherapy group. The median overall survival (OS) was not reached in either groups. The pembrolizumab group significantly prolonged OS than the chemotherapy group (HR: $0.60,95 \%$ CI: 0.41 to 0.89 ), and the objective response rate (ORR) was $44.8 \%$ vs $27.8 \%$, respectively. Treatment-related adverse events of grade 3, 4 or 5 were $26.6 \%$ and $53.3 \%$, respectively, for the two groups. Based on these findings, pembrolizumab was approved as first-line treatment for advanced NSCLC with high PD-L1 expression by the US Food and Drug Administration in October 2016. In the KEYNOTE189, a randomised, double-blind, phase III trial enrolled 616 patients with untreated metastatic non-squamous NSCLC, regardless of the PD-L1 expression level and without EGFR or ALK mutations, randomly assigned (2:1) to receive pembrolizumab or placebo plus platinum and pemetrexed regimen every 3 weeks for four cycles followed by pembrolizumab or placebo plus pemetrexed regimen every 3 weeks for up to 35 cycles. The median PFS was 8.8 months in the pembrolizumab combination group and 4.9 months in the placebo combination group. The median OS was not reached in the pembrolizumab combination group versus 11.3 months in the placebo combination group. The response rate was $47.6 \%$ and $18.9 \%$, respectively. Grade 3 or higher adverse events occurred in $67.2 \%$ and $65.8 \%$ of patients, respectively. ${ }^{10}$ Moreover, there were fewer overall side effects with ICIs than with conventional chemotherapy. ${ }^{11}$

However, although these agents cause unique immunerelated adverse events (irAEs), ${ }^{12}$ there has been a lack of head-to-head studies comparing immunotherapy (IO) monotherapy with immunotherapyplus chemotherapy (IC) as first-line treatment for advanced NSCLC. At present, indirect comparison methods are widely used for investigating competing interventions, ${ }^{13} 14$ and research has confirmed that there is no significant difference between adjusted indirect comparisons and direct comparisons. ${ }^{15}$ Thus, in the absence of direct evidence, indirect comparison methods may provide relatively valid evidence to compare two interventions. Related applications have been made for ICIs. ${ }^{16}$

This study aimed to indirectly compare the efficacy of IO monotherapy with that of IC for advanced NSCLC. Towards this goal, we conducted a systematic review of related published articles and conference reports of clinical trials. To preserve randomisation characteristics to a certain extent and minimise bias, we used the special software 'ITC' to adjust for the indirect comparison. ${ }^{17}$ Given the importance of risk-benefit analysis in decision-making related to treatment, we also analysed the adverse reactions associated with IO and IC. Furthermore, subgroup analyses of irAEs and adverse events of interest in the as-treated population were performed.

\section{METHODS}

\section{Literature search and study eligibility}

This report was prepared and written following the Preferred Reporting Items for Systematic Reviews and Meta-Analyses (PRISMA) statement. ${ }^{18}$ The retrieval time for each database was from the inception of the study to July 2019; the PubMed, Cochrane Library and Embase databases were searched for related studies on advanced NSCLC. We also searched ClinicalTrials.gov, the American Society of Clinical Oncology Meeting Library and the World Conference on Lung Cancer for related conference literature. The following keywords were used: non-small cell lung cancer, nivolumab, pembrolizumab, atezolizumab, durvalumab, avelumab, PD-1, PD-L1 and the corresponding Medical Subject Headings vocabulary. Only studies written in English were included. Simultaneously, the references of the retrieved literature were checked to further find relevant clinical trials. The inclusion criteria were as follows: (1) randomised controlled trials on the treatment of NSCLC, (2) all individuals in the studies had not received treatment previously and (3) studies on IO monotherapy using PD-1/PD-L1 inhibitors or IC. All screening and evaluation tasks were completed independently by two reviewers. Discrepancies were resolved by involving a third researcher to achieve consensus.

\section{Information extraction and quality assessment}

After reading the original literature, two reviewers independently extracted the following information: National Clinical Trial number, first author, publication year, intervention measures, number of patients in each group, phase of study, participant characteristics, tumour histology, PD-L1 expression level and cancer driver gene mutation status of NSCLC. The primary outcomes were PFS, OS and ORR. We also extracted the following data: treatment-related adverse events (TRAEs); events leading to discontinuation of treatment; events leading to death; and irAEs. The irAEs included hypothyroidism, hyperthyroidism, pneumonitis, infusion reaction, severe skin reaction, thyroiditis, colitis, hypophysitis, nephritis, pancreatitis, hepatitis and adrenal insufficiency. All adverse events were assessed and graded according to the National Cancer Institute Common Terminology Criteria for Adverse Events.

We used the 'risk of bias' method recommended in the Cochrane Handbook for Systematic Reviews of Interventions $^{19}$ to assess the methodological quality of the included studies. The following factors were assessed: 
random sequence generation, allocation concealment, blinding of participants and personnel, blinding of outcome assessment, incomplete outcome data, selective reporting and other bias. Risk assessment for bias was based on the following criteria: low risk, high risk and unclear risk. Two researchers completed the above tasks independently. Begg's test and Egger's test were used as quantitative tests for publication bias ${ }^{20}$ with the $p$ value representing the degree of bias. A $p$ value of $<0.1$ indicated publication bias. Sensitivity analysis was conducted by eliminating the included articles one by one. $^{19}$

\section{Statistical analysis}

For survival data (PFS and OS), HRs were used to represent the survival analysis of intervention effects. Analysis of ORR, TRAEs and irAEs were performed according to dichotomous data. Risk ratios (RRs) were used as value indices of effects. We also calculated the $95 \%$ CI of the corresponding indicators. Statistical heterogeneity in the included studies was evaluated using the $\chi^{2}$ test and $\mathrm{I}^{2}$ statistic. When $\mathrm{I}^{2}$ was $<50 \%$ and $\mathrm{p}$ value was $>0.1$, a fixed effects model was selected to combine the studies; $;^{19}$ otherwise, a random effects model was used, and when $\mathrm{p}$ value was $<0.05$, the difference in efficacy between different interventions was considered statistically significant. When the $95 \%$ CI for indirect comparison contained 1 , the difference was considered not statistically significant. Adjusted indirect comparisons were conducted using chemotherapy (arm C) as the common therapeutic arm. By comparing IO (arm A) with chemotherapy and IC ( $\operatorname{arm} B)$ with chemotherapy, the relative effect of IO versus IC was indirectly evaluated. ${ }^{13}$ The result of $\log$ HR was estimated using the formula $\log \mathrm{HR}_{\mathrm{AB}}=\log \mathrm{HR}_{\mathrm{AC}}-\log \mathrm{HR}_{\mathrm{BC}}$, and its $\mathrm{SE}$ was estimated as $\mathrm{SE}\left(\log \mathrm{HR}_{\mathrm{AB}}\right) \sqrt{\mathrm{SE}\left(\log \mathrm{HR}_{\mathrm{AC}}\right)^{2}+\mathrm{SE}\left(\log \mathrm{HR}_{\mathrm{BC}}\right)^{2}}$. RR was calculated in a similar manner. ${ }^{22}$ We also conducted a subgroup analysis to explore the sources of heterogeneity. According to PD-L1 expression, the main subgroup included PD-L1 high expressed subgroup ( $\geq 50 \%)$, PD-L1 low expressed subgroup ( $1 \%$ to $49 \%$ ) and PD-L1 positive subgroup $(\geq 1 \%)$. Due to lack of data, the subgroup of PD-L1 expression less than $1 \%$ was not performed. Not all the trials reported the efficacy and safety results in each subgroup. We extracted the subgroup analysis data of all the trials according to the pre-designed grouping factors, and each trial was included only once per subgroup. All statistical analyses were performed using Stata statistical software (V.12.0; StataCorp LP, College Station, Texas, USA) and ITC software (V.1.0; Canadian Agency for Drugs and Technologies in Health, Ottawa, Ontario, Canada).

\section{Patient and public involvement}

Patients and/or the public were not involved in the design, or conduct, or reporting, or dissemination plans of this research.

\section{RESULTS}

\section{Study eligibility and quality assessment}

We initially collected 723 related publications, of which 134 were derived from ClinicalTrials.gov and included conference abstracts. After removing duplicates, 556 texts were screened, and 22 articles were finally assessed for eligibility after reading the abstracts. There were six trials without a control group, and five studies had interventions including ipilimumab. One study did not include a chemotherapy group, and one study included bevacizumab in the combination therapy regimen. Finally, 10 clinical trials were included in this meta-analysis. ${ }^{9} 10$ 23-30 Figure 1 shows the flow diagram for selection of the searched literature, and table 1 presents the basic features of the selected studies. Among the 10 studies, 3 were on IO alone ( 1 on nivolumab and 2 on pembrolizumab), and 7 were on IC ( 1 on nivolumab, 3 on pembrolizumab and 3 on atezolizumab). Only 1 study was a phase II clinical trial, and the rest were phase III trials. We found 3 clinical trials for IO versus chemotherapy ( $\mathrm{n}=2120$ patients) and 7 clinical trials for IC versus chemotherapy $(n=3645$ patients). The risk of bias assessment showed that the risk was within acceptable limits (online supplemental figure $1)$.

\section{Overall survival}

The meta-analysis of OS in patients with advanced NSCLC treated with IO and IC as first-line treatment is shown in online supplemental figure 2. The HR was 0.83 (95\% CI: 0.64 to 1.08 ) in the IO group and 0.71 (95\% CI: 0.58 to 0.88 ) in the IC group. After indirect comparison (figure 2), the HR of OS between IO and IC was 1.17 (95\% CI: 0.84 to 1.63 ). There was a trend towards improved OS with IC, but the difference was not statistically significant.

We conducted a subgroup analysis for OS according to PD-L1 expression (online supplemental figure 2 and figure 2). In the high PD-L1 subgroup ( $\geq 50 \%)$, the HR was 0.71 (95\% CI: 0.60 to 0.84$)$ for IO and 0.59 (95\% CI: 0.46 to 0.77$)$ for IC. However, there was no significant difference between IO and IC (HR: 1.20, 95\% CI: 0.89 to 1.64). In the low PD-L1 subgroup ( $1 \%$ to $49 \%$ ), there was no significant difference between IO and IC (HR: 1.23, 95\% CI: 0.65 to 2.30$)$. In the PD-L1 positive subgroup $(\geq 1 \%)$, apparently, IO yielded a significantly inferior OS than IC (HR: $1.40,95 \%$ CI: 1.06 to 1.85 ).

Further subgroup analyses according to age, sex, Eastern Cooperative Oncology Group (ECOG) performance status, histological type and smoking status for OS (figure 3) showed that IC yielded a significantly superior OS than IO among female patients, especially those with an ECOG performance status of 1, those with non-squamous histology and those who never smoked. However, in the squamous NSCLC subgroup, although there was no significant difference between the IO and IC regimens (HR: $0.95,95 \%$ CI: 0.70 to 1.28 ), there was a trend towards improved OS with IO monotherapy. 


\section{Studies identified through database searching in PubMed,Embase and Cochrane Library $(n=589)$}

Additional records identified through ClinicalTrials.gov and Conferences Abstract searching $(n=134)$

Records excluded based on screening of titles and abstracts $(n=530)$ Records after duplicates removed $(n=556)$

Clinical trial is ongoing $(n=4)$

Studies excluded $(n=12)$

Single-Arm studies( $n=6)$

Contained Ipilimumab's studies $(n=5)$

Combination therapy included bevacizumab(n=1)

Studies assessed for eligibility $(n=22)$

No chemotherapy group $(n=1)$

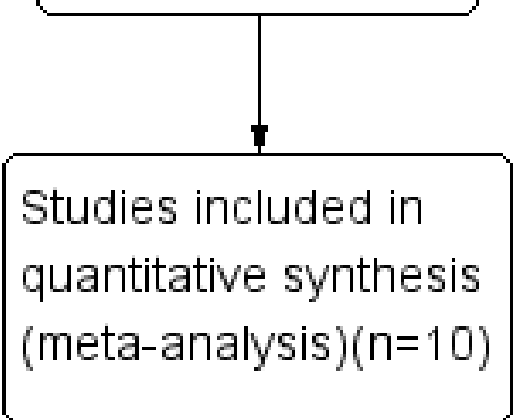

Figure 1 Flowchart for the selection of the searched literature to be included in the studies. 


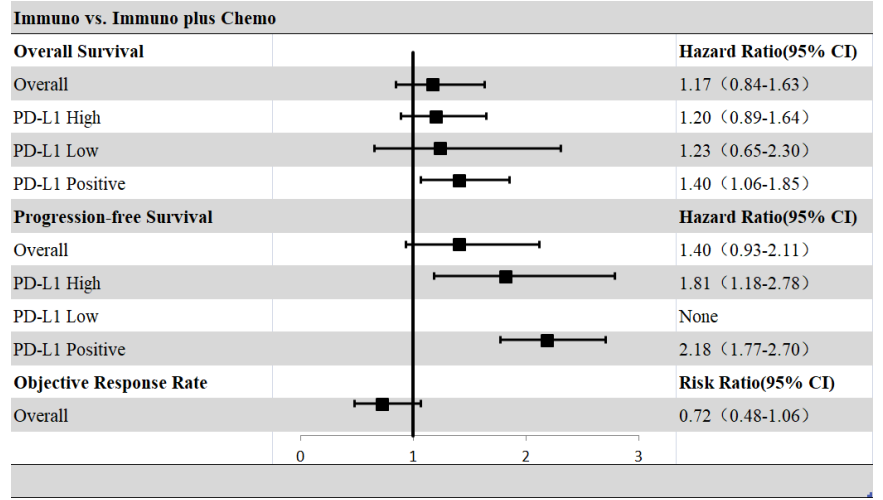

Figure 2 Forest plots of the indirect comparisons of overall survival, progression-free survival and objective response rate. Chemo,chemotherapy; Immuno, immunotherapy alone; Immuno plus chemo, immunotherapyplus chemotherapy; PDL1, programmed death ligand 1.

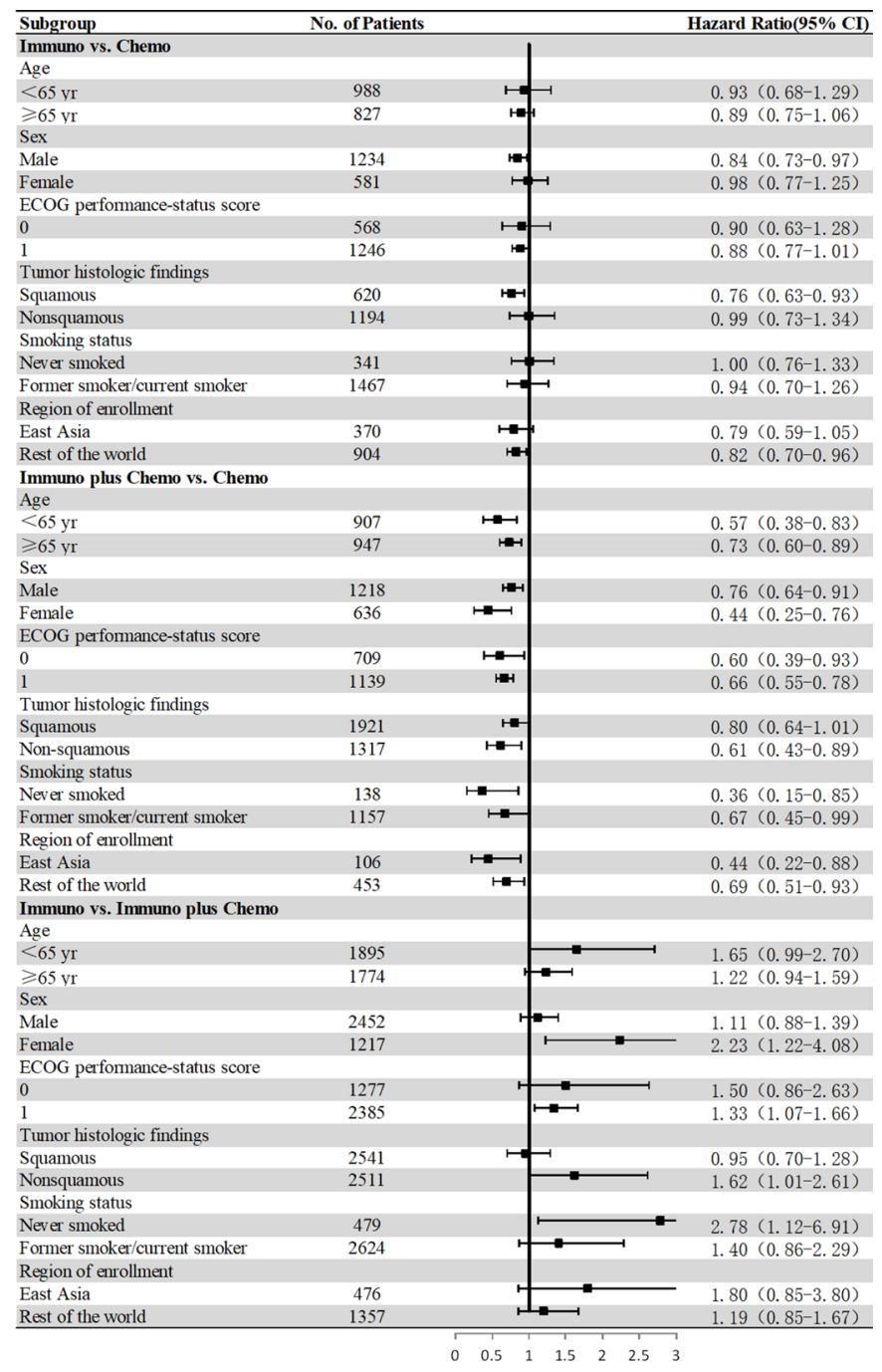

Figure 3 Forest plots of hazard ratios for overall survival according to stratification analysis. Immuno, immunotherapy alone; Chemo, chemotherapy; Immuno plus chemo, immunotherapy plus chemotherapy.
Progression-free survival

The HRs of PFS in the patients with advanced NSCLC treated with IO or IC as first-line treatment are shown in online supplemental figure 3. The HR was 0.87 (95\% CI: 0.59 to 1.30 ) for IO vs chemotherapy and 0.62 (95\% CI: $0.560 .69)$ for IC vs chemotherapy. An indirect comparison between the two therapeutic regimens showed that patients receiving IO tended to experience more progression events than those receiving IC (HR: $1.40,95 \%$ CI: 0.93 to 2.11; figure 2), although there was no significant difference between the groups.

Subgroup analysis according to PD-L1 expression (online supplemental figure 3 and figure 2) showed that IO was inferior to IC in terms of PFS (HR: $1.81,95 \%$ CI: 1.18 to 2.78 ), and the difference was statistically significant. When PD-L1 expression was at least $50 \%$, the HR of PFS was 0.76 (95\% CI: 0.52 to 1.11$)$ for IO and $0.42(95 \%$ CI: 0.34 to 0.51 ) for IC. When PD-L1 expression was at least $1 \%$, the HR of PFS for IO vs IC was 2.18 (95\% CI: 1.77 to 2.70 ) through indirect comparison.

Further subgroup analyses of PFS according to age, sex, ECOG performance status, histological type, and smoking status (figure 4) showed that IC was significantly superior to IO in female patients and never-smokers. There was a trend towards improved PFS with IO in the squamous NSCLC subgroup (HR: 0.91, 95\%: CI 0.38 to $2.14)$, whereas IO was inferior to IC in the non-squamous NSCLC subgroup (HR: 1.47, 95\% CI: 0.63 to 3.43), although there was no statistically significant difference, which is similar to that for OS.

\section{Objective response rate}

The RR of the ORR was 1.08 (95\% CI: 0.76 to 1.54 ) in the IO group and 1.51 (95\% CI 1.28 to 1.78$)$ in the IC group (online supplemental figure 4). The results showed that IO was inferior to IC as first-line treatment for advanced NSCLC (RR: 0.72, 95\% CI: 0.48 to 1.06 ) (figure 2), although there was no significant difference in ORR between the regimens.

\section{Adverse events}

The incidence of any-grade TRAEs was significantly lower in the IO group than in the IC group (RR: $0.73,95 \%$ CI: 0.66 to 0.81 ; figure 5 ). Moreover, the incidence of TRAEs was lower with IO monotherapy than with chemotherapy (RR: 0.75 , 95\% CI: 0.69 to 0.83 ), whereas it was higher with IC than with chemotherapy (RR: $1.03,95 \%$ CI: 0.99 to 1.07 ). With respect to grade 3,4 or 5 TRAEs and events leading to the discontinuation of treatment, IO was still superior to IC (RR: $0.35,95 \%$ CI: 0.29 to 0.44 and RR: $0.56,95 \%$ CI: 0.42 to 0.76 , respectively), and the differences were statistically significant. The incidences of grade 3,4 or 5 TRAEs and events leading to the discontinuation of treatment were also lower for IO than for chemotherapy (RR: $0.42,95 \%$ CI: 0.35 to 0.51 , and RR: $0.84,95 \%$ CI: 0.65 to 1.09 , respectively). Furthermore, the incidences of grade 3, 4 or 5 TRAEs and events leading to the discontinuation of treatment were higher for IC 


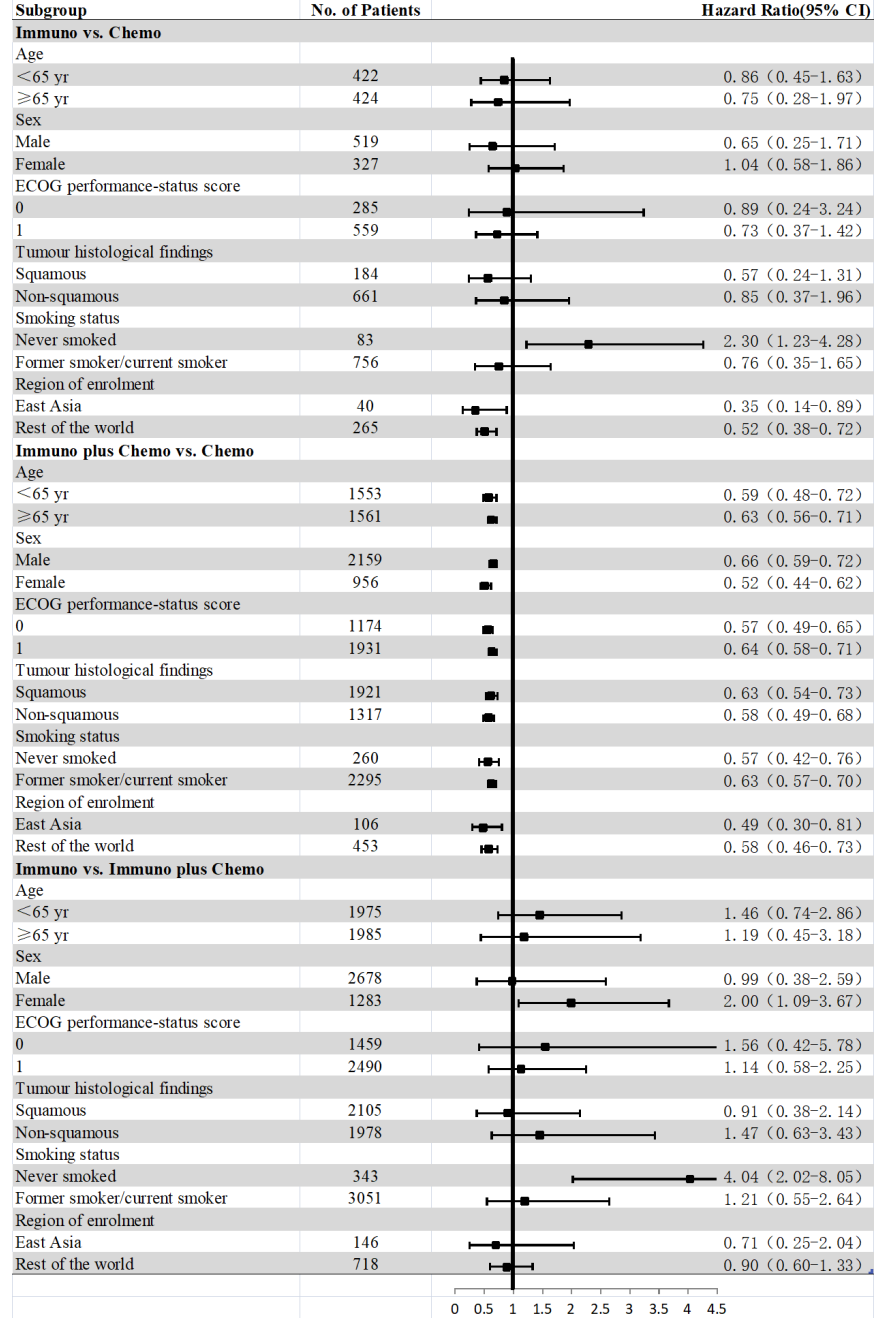

Figure 4 Forest plots of HRs for progression-free survival according to stratification analysis. Chemo,chemotherapy; ECOG, Eastern Cooperative Oncology Group;Immuno, immunotherapy alone; Immuno plus chemo, immunotherapy plus chemotherapy. than for chemotherapy (RR: 1.19, 95\% CI: 1.07 to 1.32 , and RR: $1.49,95 \%$ CI: 1.29 to 1.73 , respectively). Meanwhile, the mortality rate was similar between IO and IC (RR: $0.86,95 \%$ CI: 0.39 to 1.89 ).

Patients receiving IO alone had a significantly higher risk of irAEs than those receiving IC (figure 5) for previously untreated NSCLC (RR: 2.02, 95\% CI: 1.21 to 3.36). Pooled analysis of grade 3,4 or 5 irAEs indicated that the RR for IO versus IC was 2.12 (95\% CI: 0.99 to 4.51 ) through indirect comparison (figure 5). The incidence rates of hypothyroidism, pneumonitis, hyperthyroidism, severe skin reaction, infusion reaction, thyroiditis, hepatitis, colitis, adrenal insufficiency, hypophysitis, nephritis and pancreatitis were also analysed (table 2). The results of combined analysis showed that except for those of infusion reaction (RR: $0.20,95 \%$ CI: 0.08 to 0.53 ) and colitis (RR: 0.85 , 95\% CI: 0.14 to 4.95 ), the incidence of irAEs was higher with IO alone than with IC.

\section{Publication bias}

The publication bias assessment was performed for the RR of the ORR. Both Begg's test and Egger's test showed a $\mathrm{p}$ value of $>0.1 \quad(\mathrm{p}=0.721$ and $\mathrm{p}=0.386$, respectively $)$, suggesting no publication bias. The corresponding funnel plot is shown in online supplemental figure 5). The sensitivity analysis suggested that the included estimates were essentially within the CIs of the total effect value (online supplemental figure 6), indicating that the results were stable.

\section{DISCUSSION}

In this meta-analysis, we found that there were no significant differences in the OS, PFS and ORR between IO and IC as first-line treatment for advanced NSCLC. The results of this systematic review supported that IC as a first-line treatment for advanced NSCLC could improve PFS compared with IO when the PD-L1 expression was

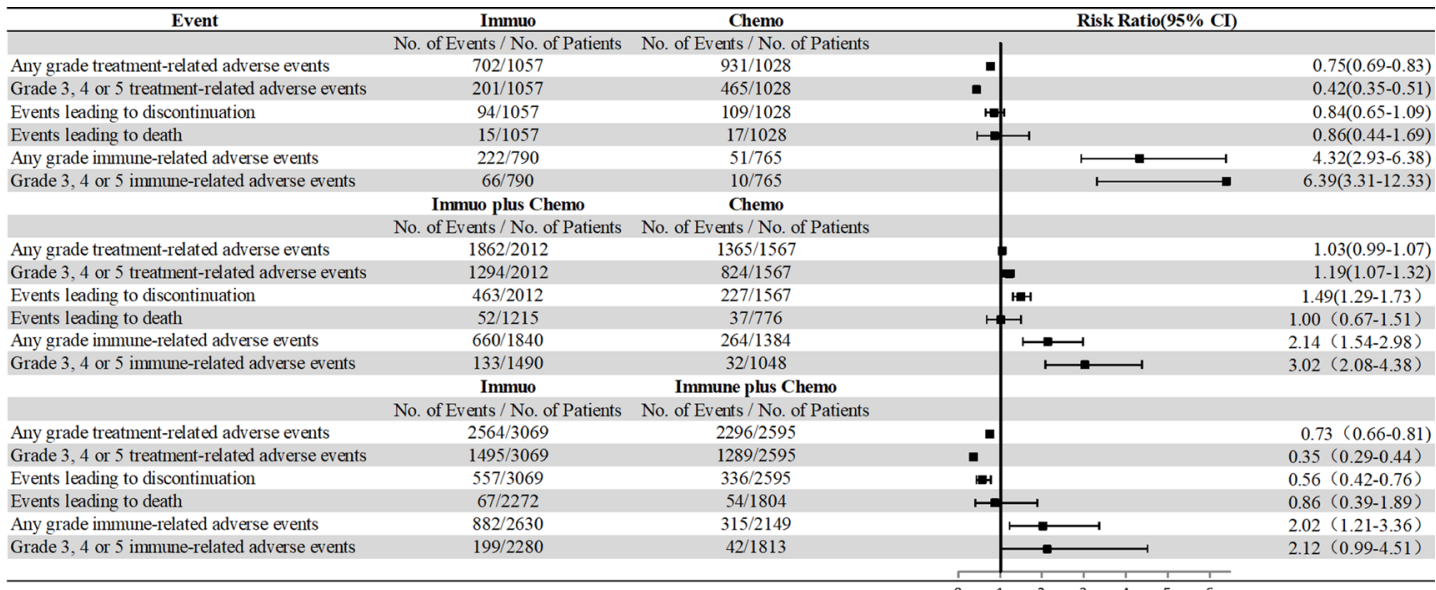

Figure 5 Forest plots of risk ratios for adverse events. Chemo,chemotherapy; Immuno, immunotherapy alone; Immuno plus chemo, immunotherapyplus chemotherapy. 


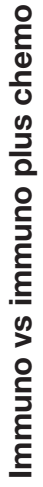

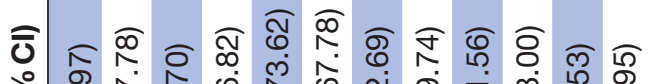

คิ่

잉ㅇㅇㅇㅇㅇㅇㅇㅇㅇㅇㅇㅇㅇ

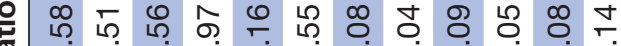

- e e e e e e e e e o

एँ

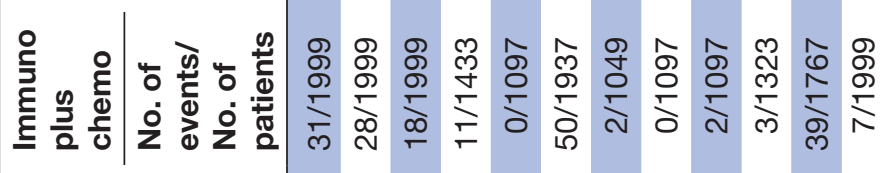

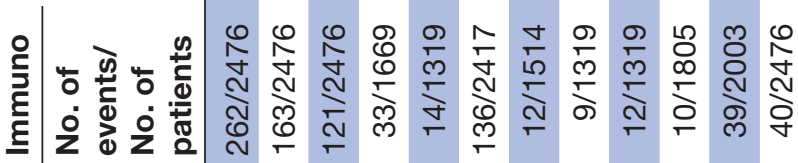

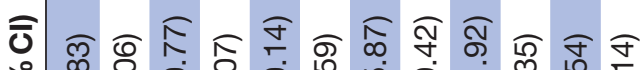

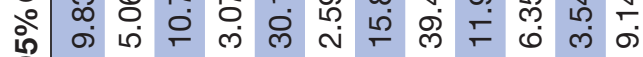

응ㅇㅇㅇㅇㅇㅇㅇㅇㅇㅇㅇㅇㅇㅇㅇㅇ 우

은 융

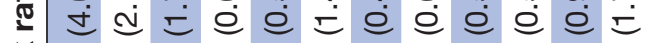

苂

द्व U.

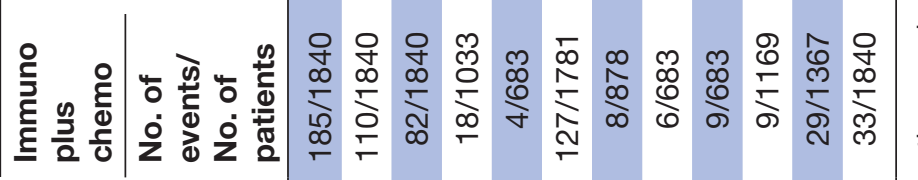

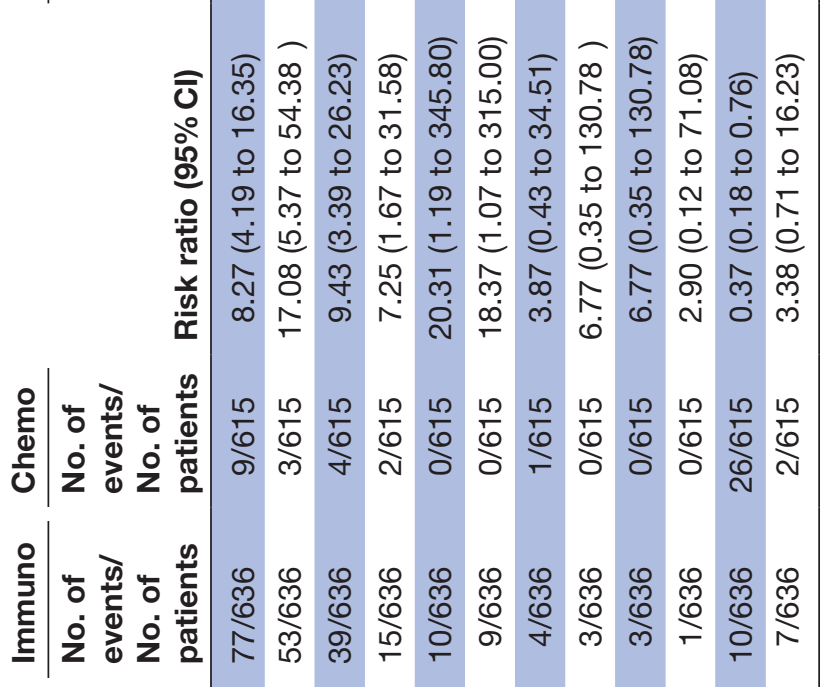

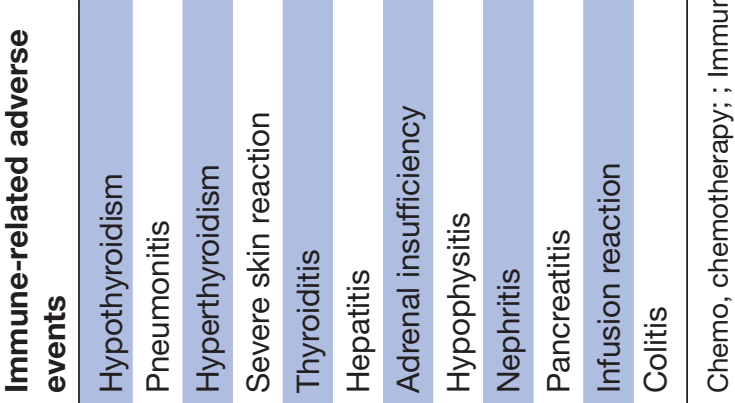


at least $50 \%$ and at least $1 \%$, providing instrumental evidence that could be used towards a more individualised treatment approach in NSCLC.

ICIs can promote the growth and proliferation of $\mathrm{T}$ lymphocytes by blocking the binding of PD-1 and PD-L1, enhancing the ability of $\mathrm{T}$ lymphocytes to recognise tumour cells, activating them to attack and kill tumour cells and achieving resistance. ${ }^{31}{ }^{32}$ When patients have tumours with high PD-L1 expression, ICIs can achieve better therapeutic effects. Studies have shown that chemotherapy can induce tumour cells to express PD-L1, which may increase the expression of PD-L1 on the surface of tumour cells. Concurrently, chemotherapeutic drugs can increase immunogenicity, regulate immune responses and enhance the ability of the immune system to recognise tumour cells. ${ }^{33}$ Zhou et al confirmed that pembrolizumab plus chemotherapy was better than pembrolizumab alone (ORR: RR=1.62, 95\% CI: 1.18 to 2.23, $\mathrm{p}=0.003$; PFS: $\mathrm{HR}=0.55,95 \%$ CI: 0.32 to 0.97 , $\mathrm{p}=0.037)$ as first-line treatment for advanced NSCLC, and a PD-L1 Tumour Proportion Score was at least 50\% using indirect comparison meta-analysis. ${ }^{34}$ However, the network meta-analysis by Doherty $e t a l^{5}$ indicated no significant differences between pembrolizumab plus chemotherapy and pembrolizumab alone as first-line treatment for PD-L1 positive advanced NSCLC in OS (HR: $0.85,95 \%$ CI: 0.45 to $1.59, \mathrm{p}=0.60$ ) and PFS (HR: $0.73,95 \%$ CI: 0.48 to $1.10, \mathrm{p}=0.13$ ). Consistent with the above research, pembrolizumab plus chemotherapy had a higher ORR than pembrolizumab alone $(+16.9 \%, 95 \%$ CI $0.7 \%$ to $33 \%, \mathrm{p}=0.04) .{ }^{35}$ There have been no comparisons of the efficacy of IO alone (more than pembrolizumab) with that of IC for advanced NSCLC. In our study, we conducted this analysis with PD-1 and PD-L1 inhibitors to expand the applications.

Furthermore, IC was significantly more beneficial than IO among female patients and never-smokers. A retrospective review indicated that never-smokers had higher chemotherapy response rates than former and current smokers. ${ }^{36}$ A published network meta-analysis supported that pembrolizumab plus chemotherapy seemed to benefit women more than men. ${ }^{37}$ At the same time, there was a considerable overlap between the subgroups stratified by sex and smoking status. This might be the reason that the subgroup analysis of never-smokers and IC (women only) was significantly better than IO.

With the recent and continuous application of ICIs, increasing attention has been paid to TRAEs and immunerelated toxicities. ${ }^{38}$ Our study found that adding chemotherapeutic drugs to the treatment regimen increased the incidence of TRAEs significantly. While ICIs activate the immune system to induce tumour resistance, they may also damage normal tissues and organs, thus causing irAEs. We found a higher incidence of irAEs with IO alone than with IC. Similarly, Kim $e t a l^{39}$ performed a network meta-analysis and reported that, as first-line treatment for advanced NSCLC, pembrolizumab plus chemotherapy had a trend to be lower than pembrolizumab in the incidence of irAEs and grade 3 to 5 irAEs (RR: 0.41, 95\% CI: 0.08 to $2.16, \mathrm{p}=0.44 .{ }^{39}$ Meanwhile, Doherty et $a l^{35}$ found that overall irAEs (risk difference, RD: $-9.1 \%$, 95\% CI: -25.8 to $7.6 \%, \mathrm{p}=0.29$ ) and grade 3 to 5 irAEs (RD: $-3.1 \%, 95 \%$ CI: $-9.1 \%$ to $2.85, \mathrm{p}=0.30$ ) tended to be lower with pembrolizumab plus chemotherapy compared with pembrolizumab alone. ${ }^{35}$ With respect to complications, the $95 \%$ CIs in both arms were wider, and the estimation of outcome indicators was not sufficiently accurate. It was therefore necessary to increase the sample size to narrow the interval width. This result should be interpreted with caution. Toxic reactions caused by therapeutic drugs may cause serious or life-threatening adverse events. Thus, the early identification of complications and administration of treatment are important. ${ }^{41}$

Subgroup analysis according to the pre-planned grouping factors showed that there were no definite factors causing heterogeneity. Furthermore, the results of the sensitivity analysis showed that the meta-analysis was statistically stable. However, it was also important to take into account the heterogeneity between studies, including the difference in the type of ICIs and platinum-based chemotherapy regimens. However, in previous studies, different chemotherapy regimens (eg, cisplatin+paclitaxel, cisplatin+gemcitabine, cisplatin+docetaxel or carboplatin+paclitaxel) for advanced NSCLC had similar therapeutic effects. ${ }^{42}$ Similarly, the KEYNOTE-407 trial indicated similar therapeutic effects between paclitaxel and nab-paclitaxel for advanced NSCLC. ${ }^{23}$ Therefore, it was reasonable to assume that these chemotherapy regimens had similar efficacy for advanced NSCLC. The baseline characteristics of each trial were similar in tumour histology, age, sex and smoking history, which supported the validity of the adjusted indirect comparisons described by the network meta-analysis. In all including studies, immunotherapy plus chemotherapy almost had a significant better OS than chemotherapy, and it might minimise the potential bias caused by the intrinsic heterogeneity.

However, this study still has some limitations. First, the study was an indirect comparison, and the level of evidence was relatively low. Hence, head-to-head clinical trials are needed to verify our findings. Second, there were fewer randomised controlled trials of first-line treatment for advanced NSCLC with current findings, which led to few studies being included in this analysis, especially for IO, and further analysis was not possible. For example, we were unable to analyse PD-1 and PD-L1 inhibitors separately. In addition, not all studies reported the outcome indicators in this meta-analysis, and the sample sizes were different between IC and IO. This might lead to the imbalance of the patient population to affect the comparability of the indirect comparison and thus produce a potential selection bias. Finally, three of the included studies were summaries of conference abstracts, some of which did not have final results. Therefore, it is necessary to establish direct comparisons between both regimens and conduct large-scale clinical trials to provide more reliable evidence for the choice of clinical treatment. 


\section{CONCLUSIONS}

For advanced NSCLC, IO is not inferior to IC as first-line treatment with respect to survival benefit, as evidenced by the lack of significant differences in OS, PFS and ORR between the two regimens. However, we found that IC had better efficacy than IO alone as first-line treatment in advanced NSCLC patients with PD-L1 expression in at least $50 \%$ and at least $1 \%$ of tumour cells. However, IC was associated with more TRAEs, whereas IO was associated with more irAEs. This difference in related adverse events highlights the need for considering the physical health and characteristics of patients for a more individualised treatment approach. In the future, head-to-head clinical studies are necessary to verify the results of our current study.

Contributors LL, FX and YC contributed to the collection and evaluation of data and statistical analysis. LL wrote the manuscript. XR and YL contributed to the interpretation of data. SX and YC contributed to the study design, statistical analysis and interpretation of results. All authors contributed to the revision of the manuscript.

Funding This work was supported by the Double First-Class University Plan funding of 2016 from Tongji Medical College, Huazhong University of Science and Technology (grant no. 5001540022) and the Hubei Provincial Health Planning Commission (grant no. WJ2017M082).

Competing interests None declared.

Patient consent for publication Not required.

Provenance and peer review Not commissioned; externally peer reviewed.

Data availability statement Data are available in a public, open access repository. Extra data can be accessed via the Dryad data repository at http://datadryad.org/ with the doi:10.5061/dryad.0gb5mkkzv.

Supplemental material This content has been supplied by the author(s). It has not been vetted by BMJ Publishing Group Limited (BMJ) and may not have been peer-reviewed. Any opinions or recommendations discussed are solely those of the author(s) and are not endorsed by BMJ. BMJ disclaims all liability and responsibility arising from any reliance placed on the content. Where the content includes any translated material, BMJ does not warrant the accuracy and reliability of the translations (including but not limited to local regulations, clinical guidelines, terminology, drug names and drug dosages), and is not responsible for any error and/or omissions arising from translation and adaptation or otherwise.

Open access This is an open access article distributed in accordance with the Creative Commons Attribution Non Commercial (CC BY-NC 4.0) license, which permits others to distribute, remix, adapt, build upon this work non-commercially, and license their derivative works on different terms, provided the original work is properly cited, appropriate credit is given, any changes made indicated, and the use is non-commercial. See: http://creativecommons.org/licenses/by-nc/4.0/.

\section{ORCID iD}

Lingling Li http://orcid.org/0000-0002-8041-4419

\section{REFERENCES}

1 Siegel RL, Miller KD, Jemal A. Cancer statistics, 2018. CA Cancer J Clin 2018;68:7-30.

2 Cheng G, Akhurst T. Lung cancer. PET Clin 2018;13:xi-xii.

3 Ettinger DS, Wood DE, Akerley W, et al. NCCN guidelines insights: non-small cell lung cancer, version 4.2016. J Natl Compr Canc Netw 2016;14:255-64.

4 Pabani A, Butts CA. Current landscape of immunotherapy for the treatment of metastatic non-small-cell lung cancer. Curr Oncol 2018;25:94-102.

5 Bansal P, Osman D, Gan GN, et al. Recent advances in immunotherapy in metastatic NSCLC. Front Oncol 2016;6:239.

6 Remon J, Pardo N, Martinez-Martí A, et al. Immune-checkpoint inhibition in first-line treatment of advanced non-small cell lung cancer patients: current status and future approaches. Lung Cancer 2017; 106:70-5

7 La-Beck NM, Jean GW, Huynh C, et al. Immune checkpoint inhibitors: new insights and current place in cancer therapy. Pharmacotherapy 2015;35:963-76.

8 Chae YK, Arya A, lams W, et al. Immune checkpoint pathways in non-small cell lung cancer. Ann Trans/ Med 2018;6:88.

9 Reck M, Rodríguez-Abreu D, Robinson AG, et al. Pembrolizumab versus chemotherapy for PD-L1-positive non-small-cell lung cancer. N Engl J Med 2016;375:1823-33.

10 Gandhi L, Rodríguez-Abreu D, Gadgeel S, et al. Pembrolizumab plus chemotherapy in metastatic non-small-cell lung cancer. $N$ Engl $J$ Med 2018;378:2078-92.

11 Nishijima TF, Shachar SS, Nyrop KA, et al. Safety and tolerability of PD-1/PD-L1 inhibitors compared with chemotherapy in patients with advanced cancer: a meta-analysis. Oncologist 2017;22:470-9.

12 Weber JS, Yang JC, Atkins MB, et al. Toxicities of immunotherapy for the practitioner. J Clin Oncol 2015;33:2092-9.

13 Glenny AM, Altman DG, Song F, et al. Indirect comparisons of competing interventions. Health Technol Assess 2005;9:1-134.

14 Li L, Catalá-López F, Alonso-Arroyo A, et al. The global research collaboration of network meta-analysis: a social network analysis. PLoS One 2016;11:e0163239.

15 Song F, Altman DG, Glenny A-M, et al. Validity of indirect comparison for estimating efficacy of competing interventions: empirical evidence from published meta-analyses. BMJ 2003;326:472.

16 Peng T-R, Tsai F-P, Wu T-W. Indirect comparison between pembrolizumab and nivolumab for the treatment of non-small cell lung cancer: a meta-analysis of randomized clinical trials. Int Immunopharmacol 2017;49:85-94.

17 Bucher HC, Guyatt GH, Griffith LE, et al. The results of direct and indirect treatment comparisons in meta-analysis of randomized controlled trials. J Clin Epidemiol 1997:50:683-91.

18 Moher D, Liberati A, Tetzlaff J, et al. Preferred reporting items for systematic reviews and meta-analyses: the PRISMA statement. PLOS Med 2009;6:e1000097.

19 Higgins JPT, Green S. Cochrane Handbook for Systematic Reviews of Interventions Version 5.1.0 [updated March 2011], 2011.

20 Egger M, Davey Smith G, Schneider M, et al. Bias in meta-analysis detected by a simple, graphical test. BMJ 1997;315:629-34.

21 Begg CB, Mazumdar M. Operating characteristics of a rank correlation test for publication bias. Biometrics 1994;50:1088-101.

22 Song F, Harvey I, Lilford R. Adjusted indirect comparison may be less biased than direct comparison for evaluating new pharmaceutical interventions. J Clin Epidemiol 2008;61:455-63.

23 Paz-Ares L, Luft A, Vicente D, et al. Pembrolizumab plus chemotherapy for squamous non-small-cell lung cancer. N Engl J Med 2018;379:2040-51.

24 Borghaei H, Langer CJ, Gadgeel S, et al. 24-Month Overall Survival from KEYNOTE-021 Cohort G: Pemetrexed and Carboplatin with or without Pembrolizumab as First-Line Therapy for Advanced Nonsquamous Non-Small Cell Lung Cancer. J Thorac Oncol 2019;14:124-9.

25 Borghaei H, Hellmann MD, Paz-Ares LG, et al. Nivolumab (Nivo) + platinum-doublet chemotherapy (chemo) vs chemo as first-line (1L) treatment (tx) for advanced non-small cell lung cancer (NSCLC) with $<1 \%$ tumor PD-L1 expression: Results from CheckMate 227. J Clin Oncol 2018;36(Suppl):9001.

26 West $\mathrm{H}$, McCleod M, Hussein M, et al. Atezolizumab in combination with carboplatin plus nab-paclitaxel chemotherapy compared with chemotherapy alone as first-line treatment for metastatic non-squamous non-small-cell lung cancer (IMpower130): a multicentre, randomised, open-label, phase 3 trial. Lancet Oncol 2019;20:924-37.

27 Jotte RM, Cappuzzo F, Vynnychenko I, et al. IMpower131: Primary PFS and safety analysis of a randomized phase III study of atezolizumab + carboplatin + paclitaxel or nab-paclitaxel vs carboplatin + nab-paclitaxel as $1 \mathrm{~L}$ therapy in advanced squamous NSCLC. J Clin Oncol 2018;36(Suppl):LBA9000.

28 Papadimitrakopoulou VA, Cobo M, Bordoni R. IMpower132: PFS and safety results with $1 \mathrm{~L}$ Atezolizumab + Carboplatin/Cisplatin + pemetrexed in stage IV non-squamous NSCLC. IASLC 19th World Conference on Lung Cancer, 2018.

29 Mok TSK, Wu Y-L, Kudaba I, et al. Pembrolizumab versus chemotherapy for previously untreated, PD-L1-expressing, locally advanced or metastatic non-small-cell lung cancer (KEYNOTE-042): a randomised, open-label, controlled, phase 3 trial. Lancet 2019;393:1819-30.

30 Carbone DP, Reck M, Paz-Ares L, et al. First-Line nivolumab in stage IV or recurrent non-small-cell lung cancer. N Engl J Med 2017;376:2415-26. 
31 Pauken KE, Wherry EJ. Overcoming T cell exhaustion in infection and cancer. Trends Immunol 2015;36:265-76.

32 Chinai JM, Janakiram M, Chen F, et al. New immunotherapies targeting the PD-1 pathway. Trends Pharmacol Sci 2015;36:587-95.

33 Galluzzi L, Buqué A, Kepp O, et al. Immunological effects of conventional chemotherapy and targeted anticancer agents. Cancer Cell 2015;28:690-714.

34 Zhou Y, Lin Z, Zhang X, et al. First-line treatment for patients with advanced non-small cell lung carcinoma and high PD-L1 expression: pembrolizumab or pembrolizumab plus chemotherapy. $\mathrm{J}$ Immunother Cancer 2019;7:120.

35 Doherty M, Seanthel DS, Amanda Putri R, et al. Pembrolizumab alone or with chemotherapy for PD-L1 positive NSCLC: a network metaanalysis of randomized trials. J Clin Oncol 2019;37(Suppl):9087.

36 Tsao AS, Liu D, Lee JJ, et al. Smoking affects treatment outcome in patients with advanced nonsmall cell lung cancer. Cancer 2006;106:2428-36. -.

37 Dafni U, Tsourti Z, Vervita K, et al. Immune checkpoint inhibitors, alone or in combination with chemotherapy, as first-line treatment for advanced non-small cell lung cancer. A systematic review and network meta-analysis. Lung Cancer 2019;134:127-40.

38 Xu C, Chen Y-P, Du X-J, et al. Comparative safety of immune checkpoint inhibitors in cancer: systematic review and network metaanalysis. BMJ 2018;363:k4226.

39 Kim R, Keam B, Hahn S, et al. First-Line pembrolizumab versus pembrolizumab plus chemotherapy versus chemotherapy alone in non-small-cell lung cancer: a systematic review and network metaanalysis. Clin Lung Cancer 2019;20:331-8. - .

40 Champiat S, Lambotte O, Barreau E, et al. Management of immune checkpoint blockade dysimmune toxicities: a collaborative position paper. Ann Oncol 2016;27:559-74.

41 Raju S, Joseph R, Sehgal S. Review of checkpoint immunotherapy for the management of non-small cell lung cancer. Immunotargets Ther 2018;7:63-75

42 Schiller JH, Harrington D, Belani CP, et al. Comparison of four chemotherapy regimens for advanced non-small-cell lung cancer. $N$ Engl J Med 2002;346:92-8. 